\title{
TArget-Responsive Subcellular Catabolism Analysis for Early-stage Antibody-Drug Conjugates Screening and Assessment
}

\footnotetext{
Hua Sang ${ }^{1,2, \dagger}$, Jiali Liu ${ }^{2, \dagger}$, Fang Zhou ${ }^{2}$, Xiaofang Zhang ${ }^{2}$, Jingwei Zhang ${ }^{2}$, Yazhong Liu ${ }^{2}$, Guangji Wang ${ }^{2, *}$, Hui $\mathrm{Ye}^{2, *}$

${ }^{1}$ Department of pharmacy, The Affiliated Hospital of Nantong University, Xisi Road \#20, Nantong, Jiangsu 226001, P.R. China, ${ }^{2}$ Key Laboratory of Drug Metabolism and Pharmacokinetics, State Key Laboratory of Natural Medicines, China Pharmaceutical University, Tongjiaxiang \#24, Nanjing, Jiangsu, 210009, P.R. China.

${ }^{\dagger}$ Authors contributed equally to this manuscript.
}

*Corresponding author:

Prof. Guangji Wang, guangjiwang@hotmail.com, Tel. 86-25-83271176,

Prof. Hui Ye, cpuyehui@cpu.edu.cn, Tel. 86-25-83271179. 


\begin{abstract}
Key events including antibody-antigen affinity, ADC internalization, trafficking and lysosomal proteolysis-mediated payload release combinatorially determine the therapeutic efficacy and safety for ADCs. Nevertheless, a universal technology that efficiently and conveniently evaluates the involvement of these above elements to ADC payload release and hence the final therapeutic outcomes for mechanistic studies and quality assessment is lacking. Considering the plethora of ADC candidates under development owing to the ever-evolving linker and drug chemistry, we developed a TArget-Responsive Subcellular Catabolism (TARSC) approach that measures catabolites kinetics for given ADCs and elaborates how each individual step ranging from antigen binding to lysosomal proteolysis affects ADC catabolism by targeted interferences. Using a commercial and a biosimilar ado-trastuzumab emtansine (T-DM1) as model ADCs, we recorded unequivocal catabolites kinetics for the two T-DM1s in the presence and absence of the targeted interferences. Their negligible differences in TARSC profiles fitting with their undifferentiated therapeutic outcomes suggested by in vitro viability assays and in vivo tumor growth assays, highlighting TARSC analysis as a good indicator of ADC efficacy and bioequivalency. Lastly, we demonstrated the use of TARSC in assessing payload release efficiency for a new Trastuzumab-toxin conjugate. Collectively, we demonstrated the use of TARSC in characterizing ADC catabolism at (sub)cellular level, and in systematically depicting whether given target proteins affect ADC payload release and hence therapeutic efficacy. We anticipate its future use in high-throughput screening, quality assessment and mechanistic understanding of $\mathrm{ADCs}$ for drug $\mathrm{R} \& \mathrm{D}$ before proceeding to costly in vivo experiments.
\end{abstract}

\title{
KEYWORDS
}

ADCs, TArget-Responsive Subcellular Catabolism (TARSC), T-DM1, therapeutic efficacy, high throughput screening, quality assessment 


\section{INTRODUCTION}

ADCs consist of monoclonal antibodies (mAbs) conjugated with cytotoxic drugs through linkers. The mAbs carry ADCs to target cells by affinity binding to antigens expressed on cell surface, and subsequently become internalized, undergo degradation followed by the release of toxic payloads within target cells. This design confers increased specificity and reduced toxicity compared to conventional pharmaceutics such as genotoxic $\operatorname{drugs}^{1-3}$.

As a novel class of therapeutics, ADCs are embracing ever-evolving technologies in linker and payload chemistry. Specifically, linkers can be classified as cleavable chemical spacers involving hydrozone, disulfide and di/tri/tetra-peptide bonds and noncleavable spacers such as thioether and pyrophosphate diester ${ }^{4,5}$. The differences in chemical stability conferred by linkers lead to varied catabolic profiles and hence ADC therapeutic efficacies in target cells or organelles. Moreover, highly potent cytotoxic agents with defined mechanisms of action such as maytansinoid and auristatin with microtubule inhibitory functions or DNA double-strand breakers, cross-linkers and alkylators are often selected as payloads ${ }^{4}$. In addition, the site of conjugation, lysine or cysteine residues, also affects ADC metabolic and pharmacokinetic liabilities ${ }^{6,7}$. Collectively, the plethora of choices offered by conjugation site, linkers and payloads confers versatile combinations, leading to currently over 100 ADCs in clinical trials and numerous ADCs as preclinical candidates ${ }^{8}$.

Therefore, the concomitant task of druggability screening and quality assessment for the large amount of ADC candidates becomes challenging, necessitating a high throughput approach that efficiently and comprehensively evaluates ADC attributes before proceeding to expensive and time-consuming in vivo experiments. Nevertheless, conventional in vitro assaying techniques such as cytotoxicity assay merely provides a final functional readout while lacking a comprehensive evaluation of how each step ranging from antigen binding, internalization, trafficking and lysosomal proteolysis-mediated payload release functions and affects payload release, and hence the observed cell killing effect ${ }^{4}$. Conventionally, each step described above has often been studied separately. For instance, internalization rate and extent can be screened by flow cytometry and visualized by fluorescent microscopy 9,10 . 
Moreover, linker stability and lysosomal proteolysis is assessed by monitoring payload release via LC-MS/MS after incubating ADCs with miscellaneous proteolytic enzymes such as cathepsin $\mathrm{B}^{11}$, rat lysosomal lysate ${ }^{12}$ and acidified S9 fractions ${ }^{13}$. Nevertheless, a holistic landscape of how each protein target or pathway affects ADC catabolism and consequently the kinetics of catabolic payload is lacking, albeit it is agreed that such information is of paramount importance in assessing ADC safety and efficacy, and allows dissecting functional molecular pathways/proteins that affect ADC catabolism.

Thus, herein we propose a TArget-Responsive Subcellular Catabolism (TARSC) approach that monitors ADC catabolites kinetics in cancer cells and organelles, and examine kinetic changes in response to targeted interferences of given proteins/pathways. We employed a commercial T-DM1 as model ADC, and measured its catabolic behaviors in both the target organelles, lysosomes, and the target cells, HER2-overexpressing BT474 cells. Then, we pharmacologically and genetically interfered with key processes involved in T-DM1 delivery and catabolism including clathrin-mediated endocytosis, endosome/lysosome transport and lysosomal cathepsin-dependent proteolysis, and recorded significantly impaired production of the toxic payloads as expected. Further, we applied the TARSC approach to evaluate the bioequivalence of a biosimilar (BS) T-DM1 with the commercial drug (CD) regarding their TARSC profiles, and found the payloads produced from the two T-DM1s displayed almost identical kinetic behaviors upon the targeted interferences of the proteins that are involved. The negligible differences of the TARSC profiles of the two T-DM1s agree with their undifferentiated therapeutic outcomes suggested by in vitro viability assays and in vivo tumor growth assays, highlighting the close association of ADC payload kinetics with therapeutic efficacy and hence the application of TARSC analysis to ADC assessment. Lastly, we demonstrated the use of TARSC in appraising linker stability for a new trastuzumab-toxin conjugate. Collectively, TARSC analysis allows us to characterize ADC catabolism at cellular and subcellular level, and systematically depicts whether the given target proteins affect ADC delivery and payload release. Such information is anticipated to support high-throughput screening, quality assessment and mechanistic understanding of ADCs in the drug R\&D pipeline with significantly reduced cost and time consumed by in vivo experiments. 


\section{EXPERIMENTAL SECTION}

\section{Chemicals and reagents.}

The commercially available Trastuzumab-DM1 (Commercial Drug, CD) was purchased from Hoffmann-La Roche Inc (Basel, Switzerland), and the BS Trastuzumab-DM1 was manufactured by a domestic pharmaceutical company (Shanghai, China) as previously detailed $^{14}$. Chloropromazine, E-64d, CA-074 methyl ester, pepstatin A, aprotinin and tris (2-carboxyethyl) phosphine hydrochloride (TCEP) were purchased from Sigma-Aldrich (St. Louis, MO, USA). Bafilomycin A1 was purchased from Selleck Chemicals (Houston, TX, USA). Ansamitocin P-3 was purchased from Medchem Express (Monmouth Junction, NJ, USA). Cell Counting Kit-8 (CCK-8) was purchased from Beyotime (Jiangsu, China). Monoclonal antibody against HER2 (Cat\# AB16901) was purchased from Abcam (Cambridge, MA, USA). Rhodamine Red ${ }^{\mathrm{TM}}-\mathrm{X}$ (RRX) AffiniPure Donkey Anti-Mouse IgG $(\mathrm{H}+\mathrm{L})$ was purchased from Jackson ImmunoResearch Laboratories Inc. (Philadelphia, PA, USA). Acetonitrile (ACN, HPLC-grade) was purchased from Merck (Darmstadt, Germany). Deionized water was prepared by Milli-Q system (Millipore Corporation, Billerica, MA, USA). All other reagents and solvents were purchased from Sigma-Aldrich (St. Louis, MO, USA) and of analytical grade.

\section{Cell culture.}

Human breast cancer cells BT474 was purchased from the American Type Culture Collection. The cells (passages 8 to 25) were cultured in RPMI 1640 medium supplemented with $10 \%$ fetal bovine serum and $100 \mathrm{U} \cdot \mathrm{mL}^{-1}$ penicillin and streptomycin (Invitrogen, Carlsbad, CA) at $37^{\circ} \mathrm{C}$ with $5 \% \mathrm{CO}_{2}$. The medium was changed every other day.

\section{Animals.}

All animal care and experimental procedures were conducted according to the National Research Council's Guidelines for the Care and Use of Laboratory Animals and were approved by the SPF Animal Laboratory of China Pharmaceutical University (Animal authorization reference number: SYXK2016-0011). Healthy female Balb/c nude mice (18-22 $\mathrm{g}$ of weight and 6-8 weeks of age) were obtained from Shanghai SLAC Laboratory Animal Co., Ltd (Shanghai, China). The mice were maintained under controlled environment 
(22-24 ${ }^{\circ} \mathrm{C}, 50-60 \%$ humidity, $12-\mathrm{h}$ light/12-h dark cycle) with ad libitum access to standard laboratory food and water.

\section{Lysosomal TArget-Responsive Subcellular Catabolism analysis.}

The lysosomal TARSC analysis of ADCs was conducted by first incubating the ADCs with crude lysosome fractions (CLF). The CLF derived from rat liver was prepared according to the manufactures' instructions using a Lysosome Isolation Kit (Sigma-Aldrich, St. Louis, MO, USA). Lysosomal activity of prepared CLF was confirmed by the Acid Phosphatase Assay Kit (Sigma-Aldrich, St. Louis, MO, USA). The incubation was carried out at $37{ }^{\circ} \mathrm{C}$ in a final volume of $100 \mu \mathrm{L}$ containing $15 \mu \mathrm{L}$ of CLF, $75 \mu \mathrm{L}$ of $50 \mathrm{mM}$ ammonium acetate buffer (pH 5.0, with $2 \mathrm{mM}$ TCEP) and $10 \mu \mathrm{L}$ of ADC prepared in $100 \mathrm{nM}$ for $0,1,3,6,12$, 24, 48, 72, 96 and 120 hours. After incubation for different duration, the resultant ADC catabolites was determined by quantitative analysis described below, and the kinetic changes of ADC catabolites abundance were plotted against incubation time.

To perform the lysosomal TARSC analysis, we specifically examined the contribution of given lysosomal enzymes to ADC payload release by incubating protease inhibitors with the ADCs in CLF followed by quantitative analysis of the produced catabolites. The assayed protease inhibitors include cysteine protease inhibitors (CA-074-ME and E64d at $10 \mu \mathrm{M}$ ), a serine protease inhibitor (aprotinin at $20 \mu \mathrm{g} / \mathrm{mL}$ ) and an aspartic protease inhibitor (pepstatin A at $10 \mu \mathrm{g} / \mathrm{mL})$. The inhibitions were terminated by adding $400 \mu \mathrm{L}$ of ice-cold acetonitrile containing $200 \mathrm{ng} \cdot \mathrm{mL}^{-1}$ internal standard (IS). The mixtures were vortexed thoroughly, centrifuged at 30,000 g for $10 \mathrm{~min}$. The supernatants were collected, dried and reconstituted in water with $0.1 \%$ formic acid for subsequent LC-MS/MS analysis.

\section{TArget-Responsive Subcellular Catabolism analysis}

BT474 cells were treated with a CD T-DM1 and a BS T-DM1 at different concentrations $(12.5,25,50 \mathrm{nM})$, respectively. At different intervals post-administration, BT474 cells were rinsed with ice-cold PBS and lysed by three freeze-thaw cycles. Protein concentrations were measured by the Bradford assay, and the concentration of the main catabolites of T-DM1, DM1 and lys-MCC-DM1, were determined by LC-MS/MS (described below).

To conduct the TARSC analysis, given proteins involved in ADC delivery and payload release in cells were interfered by siRNA or specific inhibitors. Specifically, HER2 was 
silenced by transfecting the BT474 cells with siRNA according to the procedures described below in HER2 Knockdown. Moreover, clathrin-mediated internalization was inhibited by pre-incubating the BT474 cells with chlorpromazine at $10 \mu \mathrm{M}$ for 2 hours, and $\mathrm{H}^{+}$-ATPase activity was impaired by pre-administering $1 \mathrm{nM}$ Bafilomycin A1 to cells for 2 hours. For lysosomal proteases, $3 \mu \mathrm{M}$ CA-074-ME and $20 \mu \mathrm{M}$ E64d were pre-administered for 2 hours to inhibit cysteine protease activity, whereas $20 \mu \mathrm{g} / \mathrm{mL}$ aprotinin and pepstatin A were pre-administered for 2 hours to inhibit the proteolysis mediated by serine and aspartic protease in BT474 cells, respectively. The resultant changes of toxic payloads following the targeted interferences were quantified by LC-MS/MS.

\section{LC-MS/MS-based quantitative analysis of T-DM1 payloads}

The major catabolites of T-DM1, lys-MCC-DM1 and DM1, present in lysosomes, cell lysates and tumor mass were prepared and analyzed on a Shimadzu LC-20 HPLC system (Kyoto, Japan) coupled to 5500 QTRAP (SCIEX, Birmingham, MA, USA). Briefly, cells and tissue homogenates were protein-precipitated with 4 times volume of ice-cold ACN containing $200 \mathrm{ng} \cdot \mathrm{mL}^{-1}$ ansamitocin P-3 (IS). After centrifugation $\left(30,000 \mathrm{~g}, 10 \mathrm{~min}, 4{ }^{\circ} \mathrm{C}\right)$, the supernatants were collected and evaporated to dryness followed by reconstitution in 100 $\mu \mathrm{L}$ acetonitrile-water $(1: 1, \mathrm{v} / \mathrm{v})$. The reconstituted samples were centrifuged at $30,000 \mathrm{~g}$ for 10 min at $4{ }^{\circ} \mathrm{C}$ before injections onto the LC-MS/MS system.

For analysis of T-DM1 catabolites, chromatographic separation was conducted using a Luna $\mathrm{C}_{18}$ column $\left(100 \times 2.0 \mathrm{~mm}, 2.6 \mu \mathrm{m}\right.$, Phenomenex, Torrance, $\mathrm{CA}$, USA) at $40{ }^{\circ} \mathrm{C}$ on the Shimadzu HPLC. The mobile phase consisted of solvent A ( $0.1 \%$ aqueous formic acid) and solvent B (acetonitrile with $0.1 \%$ formic acid), and a 11-min gradient was used: $0 \mathrm{~min}, 20 \%$ B; $0.5 \mathrm{~min}, 20 \% \mathrm{~B}$; $5.5 \mathrm{~min}, 100 \% \mathrm{~B}$; $8.5 \mathrm{~min}, 20 \% \mathrm{~B}$; $11 \mathrm{~min}, 20 \% \mathrm{~B}$ with a flow rate of 0.2 $\mathrm{mL} \cdot \mathrm{min}^{-1}$. Following separation, QTRAP 5500 was operated in the positive electrospray ionization (ESI) mode for the quantitative analysis of T-DM1 payloads. Briefly, the multiple reaction monitoring (MRM) parameters employed on the QTRAP were set as follows: declustering potential set at $60 \mathrm{~V}$ for lys-MCC-DM1, DM1 and IS, collision energy set at 55 $\mathrm{eV}$ for lys-MCC-DM1, $33 \mathrm{eV}$ for DM1 and $40 \mathrm{eV}$ for the IS, MRM transitions set as $m / z$ $1103.7 \rightarrow 485.2$ for lys-MCC-DM1, $m / z 738.5 \rightarrow 547.4$ for DM1, $m / z 635.5 \rightarrow 547.3$ for the IS. Data acquisition and analysis were both performed using Analyst TF 1.5.1 software (SCIEX, 
Birmingham, MA, USA) as previously described ${ }^{15}$.

\section{HER2 knockdown}

HER2 was silenced by transfecting the BT474 cells upon plating with $10 \mathrm{nM}$ small interfering RNA (siRNA) (5'-GGA CAC GAU UUU GUG GAA Gtt-3') or 10 nM scrambled siRNA using Lipofectamine ${ }^{\circledR}$ RNAiMAX (Life Technologies, Carlsbad, CA, USA). After 72 hours of transfection, HER2 silencing efficiency was confirmed by flow cytometry and immunofluorescence staining. Briefly, cells administered with scrambled siRNA or siRNA targeting HER2 were fixed with 4\% polyformaldehyde solution. After washing and blocking with $5 \%$ bovine serum albumin, cells were incubated with anti-HER2 (1:100) at $4{ }^{\circ} \mathrm{C}$ overnight and then incubated with Rhodamine-conjugated secondary antibody (1:200) at $37{ }^{\circ} \mathrm{C}$ for 1 hour. Following additional washing, the fluorescently-labeled cells were loading onto BD Accuri ${ }^{\mathrm{TM}}$ C6 plus flow cytometer (San Jose, CA, USA) for analysis and imaged with an Olympus FV3000 confocal microscope (Shinjuku, Japan).

\section{In vitro cell growth inhibition assay}

BT474 cells was exposed to a series of concentrations of T-DM1 $(0.01,0.05,0.2,1,2.5$, $4,10,25,100 \mathrm{nM}$ ) for 120 hours at $37^{\circ} \mathrm{C}$ with $5 \% \mathrm{CO}_{2}$. After treatment, cell viabilities were measured using a CCK-8 Assay Kit and quantified by comparing viabilities with those in absence of T-DM1 exposure. IC $_{50}$ values were calculated via GraphPad Prism 7 (GraphPad Software Inc., San Diego, CA, USA).

\section{In vivo tumor growth assay}

To evaluate the in vivo therapeutic efficacy for the assayed T-DM1s, a cell line-derived xenograft $(\mathrm{CDX})$ model was established. The xenografts were generated by subcutaneous injections of $5 \times 10^{6}$ exponentially growing BT474 cells into the right flank of nude mice. Then, the mice bearing BT474 subcutaneous tumors were randomly assigned to the following three groups and administered with single-dosed T-DM1. The three groups include the control group that were injected with saline $\left(0.01 \mathrm{~mL} \cdot \mathrm{g}^{-1}, i . v\right.$. $)$, the CD T-DM1 group that were injected with the CD T-DM1 $\left(50 \mathrm{mg} \cdot \mathrm{kg}^{-1}, i . v\right.$. $)$ and the BS T-DM1 that were injected with BS T-DM1 (50 $\mathrm{mg} \cdot \mathrm{kg}^{-1}$, i.v.). Tumor volume was measured every day post-administration for each animal. On day 1, 3 and 7 post-administration, the mice were sacrificed and the tumor masses were collected. Tumor masses was weighed and 
homogenized with deionized $\mathrm{H}_{2} \mathrm{O}$ to concentrations approximately at $0.1 \mathrm{~g} / \mathrm{mL}$. Then, the homogenates were further processed as described above in the LC-MS/MS sample preparation section. The intra-tumoral concentrations of lys-MCC-DM1 and DM1 were also quantified as described above.

\section{Data analysis.}

All data are presented as mean \pm standard error of mean (SEM). Statistical analyses were performed using GraphPad Prism 7 software. Each continuous variable was analyzed for a normal distribution using the Kolmogorov-Smirnov test, and then statistical analysis was performed using a two-tailed Student's t-test or one-way ANOVA assay with Dunnett post-hoc test if $F$ was less than 0.05 and there was no significant variance inhomogeneity. Differences were considered significant at $* \mathrm{p}<0.05, * * \mathrm{p}<0.01, * * * \mathrm{p}<0.001$.

\section{RESULTS AND DISCUSSION}

\section{Development of the in vitro TARSC analysis approach for ADCs}

TARSC analysis measures the kinetic profiles of payload release by LC-MS/MS in target cells or subcellular compartments, and further probes the changes in response to targeted interference of essential proteins involved in given processes such as antigen-mAb recognition, ADC delivery and lysosomal proteolysis (Fig.1). The induced changes in payloads kinetics thus demonstrate the involvement of the assayed process in ADC catabolism and can ultimately predict their influence on ADC therapeutic outcomes. This capability has thus warranted diverse applications for TARSC analysis. First, TARSC opens new avenues for ADC quality assessment. Specifically, the acquired TARSC profiles should hold constant for ADCs of identical physicochemical attributes and hence display undistinguishable catabolic behaviors in target cells and subcellular compartments (Fig. 2-4). This will be a valuable addition to current evaluation system that judges bioequivalence between commercial drug and biosimilars or ADCs across different batches, for instance, by cytotoxicity (Fig. 5). Besides, TARSC analysis supports mechanistic studies and ADC screening. It examines which protein/process contributes to ADC catabolism and hence the cell killing effect (Fig. 6), and provides clues for further optimization, such as by swapping $\mathrm{mAb}$ or linker, when the anticipated therapeutic efficacy cannot be achieved. 


\section{Benchmarking lysosomal TARSC analysis using T-DM1s}

We first tested the use of TARSC using the commercial T-DM1 as a model ADC. Since the anti-tumoral effect of T-DM1 is exerted upon lysosomal proteolysis via payload release 16-18, the catabolism kinetics of the CD T-DM1 in lysosomes were measured. We initially employed CLF isolated from rat liver and incubated the CD T-DM1 with the CLF for 0, 1, 3, 6, 24, 48, 72, 96 and 120 hours. Sensitive detection of major T-DM1 catabolites was achieved by MRM. As shown in Fig. 2A, exposure of the CD T-DM1 to CLF delivered a time-dependent production of the catabolite lys-MCC-DM1 spanning from the entire monitored duration (1-120 hours). In contrary, another catabolite that is produced independent of lysosomal degradation and usually observed due to unstable linker chemistry, DM1, was absent, indicating that the examined TDM1 remains stable without unexpected shedding of payloads in the biological environment of CLF. This is further demonstrated by the significantly large AUC of lys-MCC-DM1 over DM1 following T-DM1 incubation (Fig. 2B).

Next, we sought to investigate whether the TARSC analysis allows for determining the lysosomal protease that contributes to T-DM1 catabolism. We incubated the CLF with inhibitors targeting different lysosomal proteases including cysteine protease, aspartic proteases and serine protease ${ }^{19,20}$, and monitored the post-inhibition kinetic profiles of the two catabolites. We found that administration of cysteine proteases inhibitors, CA-074-ME and E64d, with T-DM1 both significantly reduced the lysosomal concentration of lys-MCC-DM1 when the catabolites were collected at 24, 48, 72 and 96 hours. In comparison, the treatment with the inhibitors of aspartic proteases exhibited no changes until 96 hours and serine protease conferred no significant changes to the production of lys-MCC-DM1 at all assayed time points. The distinct outcomes induced by different protease inhibitors are in line with previous knowledge that T-DM1 is cleaved and degraded mainly dependent on cysteine $\operatorname{proteases}^{21}$.

Besides elucidating the contribution of given lysosomal proteases to ADC catabolism, we further conducted lysosomal TARSC analysis using a BS T-DM1 and compared the catabolic profiles, AUC and the changes in response to targeted interferences of lysosomal 
protease with that of the CD T-DM1. Both CD and BS T-DM1s displayed no statistical differences, revealing the bioequivalence of the two ADCs within CLF.

Previously, in vitro studies that aimed to dissect the roles of lysosomal protease in the process of ADC catabolism were conducted using different model systems including enriched lysosomes ${ }^{16}$, liver S9 fraction ${ }^{13}$ and purified lysosomal protease ${ }^{22}$. However, enzymes contained in liver S9 fractions are much more complicated than lysosomal enzymes, and the microenvironment in liver $\mathrm{S} 9$ fractions at $\mathrm{pH} 7.4$ markedly differs from the acidic $\mathrm{pH}$ in lysosomes ${ }^{13,23,24}$. Regarding the purified lysosomal proteases, numerous lysosomal enzymes would be required to truly mimic the multifarious proteolysis activities that occur within lysosomes ${ }^{25}$. Alternatively, we have shown that the easily-harvested CLF from rat liver can be exploited to monitor the ADC catabolic behaviors in general, and demonstrated to generate lys-MCC-DM1 in a time-dependent manner as those in lysosomes within the target cells BT474. Therefore, the CLF model is useful for initial screening and quality assessment of ADCs in future studies.

\section{TARSC analysis of T-DM1s empowers mechanistic studies and bioequivalence assessment}

After proving TARSC analysis enables ADC catabolism studies in lysosomes, we next asked whether TARSC is applicable to examine the released payloads from ADCs in intact cells where multiple crucial biological processes including antigen binding, intracellular internalization, trafficking and lysosomal degradation can be reflected ${ }^{10,26}$. We first measured T-DM1 payloads after the CD T-DM1 administration in BT474 cells. As shown in Fig. 3A, intracellular lys-MCC-DM1 displayed a time-dependent increase, phenocopying the trend of the catabolite, lys-MCC-DM1, produced in CLF. Compared to lys-MCC-DM1, DM1 was present at a pronounced lower concentration upon T-DM1 treatment (Fig. 3A). Moreover, its intracellular concentration and hence its AUC both exhibited a dose-response increase when T-DM1 was administered at 12.5, 25 and $50 \mathrm{nM}$, whereas that of lys-MCC-DM1 held constant (Fig. 3B). This distinction in cellular catabolic behaviors can be explained by that the amount of T-DM1 that become internalized and then catabolized to lys-MCC-DM1 reaches saturation at the lowest dose when the amount of available HER2 on cell surface are 
fully occupied by T-DM1, whereas DM1 shed from T-DM1 is directly correlated to the administered dose independent of ADC internalization rate. Further, we compared the kinetic profiles of the two T-DM1 catabolites for the CD and BS T-DM1s, and found they both exhibited unequivocal behaviors in target cells BT474 (Fig. 3A-B).

Although the above studies have shown the rate and degree of payload released from ADC degradation, it cannot dissect the role of each protein/pathway plays to confer the final therapeutic outcomes. We next applied the TARSC approach to determine how the biological cascades initializing from internalization to transport and degradation affect ADC payload kinetics. As antigen binding initializes ADC internalization and subsequent catabolism ${ }^{27}$, we first treated the BT474 cells with HER2 siRNA to suppress the HER2 expression. As shown in Fig. 4A, we noted a $48.7 \%$ decrease in HER2 expression and concomitantly the reduced concentration of lys-MCC-DM1 in cells, indicating disrupted antigen binding leads to impaired ADC payload release. We also tested whether the TARSC analysis can be applied to assess the bioequivalence of ADCs, and thus compared the TARSC profile between the CD and BS T-DM1s. Fig. 4B shows the reduction of intracellular lys-MCC-DM1 exhibited no significant difference between the two, suggesting that HER2 silencing modulated ADC delivery and catabolism to similar extents.

Next, since the internalization of cell-surface bound antigen-antibody complex is induced upon antigen binding, we then treated cells with a clathrin inhibitor, chlorpromazine, to interfere with T-DM1 internalization. In line with the clathrin-mediated trastuzumab endocytosis $^{28}$, Fig. 4C shows that intracellular concentration of lys-MCC-DM1 following the chlorpromazine treatment significantly decreased, and the concentration was reduced to comparable levels for both the CD and BS T-DM1-treated cells. Once T-DM1 enters the target cells, it is transported in endosomes to lysosomes. $\mathrm{H}^{+}$-ATPase is a proton pump present in endosomes and lysosomes, and participates in endosome-lysosome fusion ${ }^{29,30}$. After co-administration of a specific $\mathrm{H}^{+}$-ATPase inhibitor, bafilomycin $\mathrm{A} 1$, with T-DM1, the aberrant activity of $\mathrm{H}^{+}$-ATPase in lysosomes of BT474 resulted in defected transport of T-DM1. Expectedly, the released lys-MCC-DM1 from both the CD and BS T-DM1s was reduced to similar extent (Fig. 4D). Lastly, the T-DM1, once transported into lysosomes, undergoes proteolytic degradation and releases the toxic payloads to induce cancer cell 
apoptosis. In line with the lysosomal TARSC results, the production of the major catabolite, lys-MCC-DM1, was severely impaired after incubation with the cysteine protease inhibitor E64D (Fig. 4E). The primary contribution of cysteine protease to T-DM1 catabolism is further supported by the marked decrease of lys-MCC-DM1 when another cysteine protease inhibitor CA-074-ME was administered. In contrast, neither pepstatin A nor aprotinin can alter the amount of lys-MCC-DM1 catabolized from T-DM1 within 72 hours post drug administration. Further, the indistinguishable catabolic behaviors of the CD and BS T-DM1s in their target cells, and with specific interferences made to given proteins involved in antigen binding, internalization, transport and degradation, indicate that the catabolism of the two ADCs are mediated via identical pathways and are hence "bioequivalent" according to TARSC analysis.

\section{TARSC analysis predicts in vitro and in vivo therapeutic efficacy}

The effectiveness of an ADC can only be exerted when its loaded drug is released in its target cells ${ }^{31}$. Therefore, ADC's catabolic behavior is highly correlated to its therapeutic outcomes. Based on the resemblance of TARSC profiles between the CD and BS T-DM1s, we hence infer that the two types of T-DM1 should possess similar cytotoxicity against BT474 cells. This hypothesis was confirmed in Fig. 5A, which shows no statistical significance between the $\mathrm{IC}_{50}$ values of the $\mathrm{CD}$ and $\mathrm{BS}$ T-DM1s (Fig. 5B). Furthermore, to examine whether the catabolic behaviors of the two T-DM1s revealed by lysosomal and cellular TARSC study can be translated to in vivo efficacy, we performed a $15 \mathrm{mg} / \mathrm{kg}$ single-dose intravenous administration of CD or BS T-DM1 in BT474 xenograft-bearing nude mice and compared their drug catabolism profiles and the anti-tumoral effect.

Fig. 5C indicates that both CD and BS T-DM1s exerted significant suppressive effect on tumor growth after 4 days post-treatment. At 7 days post-administration, tumor volumes in the two T-DM1 treated groups have shrunk $28.6 \%$ and $38.8 \%$ compared to the volumes in the control group. No significant difference of the anti-tumor efficacy was observed between the CD and BS T-DM1s treatment.

Lastly, we sought to confirm whether T-DM1 is catabolized in vivo in similar manner as shown by in vitro TARSC analysis. We found that lys-MCC-DM1 is also the major T-DM1 
catabolite in tumor mass compared to DM1, and its AUC reaches 30.73-fold and 28.38-fold higher than the AUC of DM1 at 7 days post-treatment using the CD and BS T-DM1s, respectively (Fig. 5D). Moreover, in agreement with the negligible difference in the anti-tumoral effect observed in vivo, both CD and BS T-DM1s showed no significant difference in the AUC of lys-MCC-DM1 from the BT474 xenografts. It is thus reasonable to anticipate that the application of TARSC analysis to ADC screening is useful for inferring their therapeutic effects in vitro and in vivo, and will significantly reduce the need to perform certain in vivo pharmacological experiments for the plethora of ADC candidates during an early developmental stage.

\section{TARSC analysis for quality assessment of novel ADCs}

With the use of TARSC analysis in evaluating the bioequivalence for the CD and BS T-DM1s, we next applied TARSC to quickly assess the payload release efficiency for a new trastuzumab-toxin conjugate. A tubulysin ADC-candidate (DX-006) was designed as a cysteine-conjugated noncleavable ADC (Fig. 6A). The linker and warhead were first linked together as compound T (Tub-006) and then conjugated to cysteine residues on Trastuzumab. The prerequisite for DX-006 to induce significant cancer cell killing effect is effective toxin release upon lysosomal proteolysis. Therefore, we conducted lysosomal TARSC analysis by incubating DX-006 with the CLF and measuring the temporal changes of its major catabolites including cys-compound $\mathrm{T}$ and compound T. As shown in Fig. 6B, we noted both catabolites exhibited a time-dependent increase with prolonged incubation. Moreover, the production of compound $\mathrm{T}$ even displayed a steeper increase at later time points compared to cys-compound $\mathrm{T}$. This finding suggests rapid and efficient release of the two payloads for DX-006 (Fig. 6C).

We further pursued the catabolism route for these two products, and incubated DX-006 with protease inhibitors. Parallel to other cysteine-conjugated noncleavable ADC, cysteine protease inhibitors blocked DX-006 lysosomal metabolism and yielded decreased concentration of cys-compound $\mathrm{T}$ detected in lysosomes (Fig. 6D), indicating that lysosomal cysteine proteases are involved in the catabolic process of DX-006. Unlike cys-compound T, the administration of cysteine proteases inhibitors exhibited negligible influences on the 
concentration of compound $\mathrm{T}$, revealing the possibility that compound $\mathrm{T}$ is mainly produced in lysosomes due to Retro-Michael addition independent of the examined proteases. Together, the above TARSC results revealed the active release of payloads for the new Trastuzumab toxin conjugate in CLF, which can potentially lead to improved cytotoxic activity. Moreover, it demonstrates the use of TARSC in supporting early-stage screening of ADC candidates in high throughput before proceeding to in vivo assays.

\section{CONCLUSION}

ADCs are embracing an emerging development over the past decade. Versatile combinations of $\mathrm{mAb}$, linker and drug payload have rendered the ADC technology as a powerful drug R\&D paradigm that can efficiently reach its target cells with reduced toxicity. Nevertheless, a universal technology that efficiently and conveniently evaluates the involvement of key biological processes in ADC delivery and payload release for mechanistic studies and quality assessment is lacking. Herein, we reported the TARSC approach that measures kinetics of ADC catabolism in target cells and subcellular compartments, and records the changes when given proteins/pathways are genetically or pharmacologically interfered. TARSC can thus be applied to elucidate which proteins such as cysteine or aspartic protease contributes to ADC delivery and degradation for mechanistic studies. Moreover, we demonstrated TARSC analysis can deeply and comprehensively examine the bioequivalence for a ADC biosimilar compared to the commercial drug. Lastly, the application of lysosomal TARSC analysis to a novel Trastuzumab-drug conjugate suggests the active payload release of the assayed ADC candidate during ADC candidate screening. Collectively, we anticipate wide and versatile uses of TARSC analysis in ADC early-stage screening, assessment and mechanistic studies, and its strong support to future pharmacological investigations and medicinal chemistry design/optimization of ADCs.

\section{Acknowledgments}

We acknowledge the financial support of the National Key R\&D Program of China (2018YFD0901101), the Innovative Research Groups of the National Nature Science Foundation of China (81421005), the National Natural Science Foundation of China 
(81872838, 81803625), the Natural Science Foundation of Jiangsu Province (BK20180079, BK20180558), the Project for Major New Drug Innovation and Development (2018YFD0901101 to H.Y.), Double-First Class Initiative Project (CPU2018GY09), China Postdoctoral Science Foundation (2018M642374), Jiangsu Postdoctoral Grant Program (2018Z061), Nantong Science and Technology Project (JC2019133, JCZ18131) and the Innovation Team of Affiliated Hospital of Nantong University (TFCT-A05).

\section{References}

1 Peters, C.Brown, S. Antibody-drug conjugates as novel anti-cancer chemotherapeutics. Biosci Rep. $2015,35$.

2 Yan, H.;Endo, Y.;Shen, Y.;Rotstein, D.;Dokmanovic, M.;Mohan, N.;Mukhopadhyay, P.;Gao, B.;Pacher, P.Wu, W. J. Ado-Trastuzumab Emtansine Targets Hepatocytes Via Human Epidermal Growth Factor Receptor 2 to Induce Hepatotoxicity. Mol Cancer Ther. 2016, 15, 480-490.

3 Chari, R. V.;Miller, M. L.Widdison, W. C. Antibody-drug conjugates: an emerging concept in cancer therapy. Angew Chem Int Ed Engl. 2014, 53, 3796-3827.

4 Chalouni, C.Doll, S. Fate of Antibody-Drug Conjugates in Cancer Cells. J Exp Clin Cancer Res. 2018, 37, 20.

5 Bargh, J. D.;Isidro-Llobet, A.;Parker, J. S.Spring, D. R. Cleavable linkers in antibody-drug conjugates. Chem Soc Rev. 2019, 48, 4361-4374.

6 Hedrich, W. D.;Fandy, T. E.;Ashour, H. M.;Wang, H.Hassan, H. E. Antibody-Drug Conjugates: Pharmacokinetic/Pharmacodynamic Modeling, Preclinical Characterization, Clinical Studies, and Lessons Learned. Clin Pharmacokinet. 2018, 57, 687-703.

7 Lee, M. V.;Kaur, S.Saad, O. M. Conjugation Site Influences Antibody-Conjugated Drug PK Assays: Case Studies for Disulfide-Linked, Self-Immolating Next-Generation Antibody Drug Conjugates. Anal Chem. 2020, 92, 12168-12175.

8 Chau, C. H.;Steeg, P. S.Figg, W. D. Antibody-drug conjugates for cancer. Lancet. 2019, 394, 793-804.

9 Li, F.;Ulrich, M.;Jonas, M.;Stone, I. J.;Linares, G.;Zhang, X.;Westendorf, L.;Benjamin, D. R.Law, C. L. Tumor-Associated Macrophages Can Contribute to Antitumor Activity through FcgammaR-Mediated Processing of Antibody-Drug Conjugates. Mol Cancer Ther. 2017, 16, 1347-1354.

10 Lv, C.;Yang, C.;Ding, D.;Sun, Y.;Wang, R.;Han, D.Tan, W. Endocytic Pathways and Intracellular Transport of Aptamer-Drug Conjugates in Live Cells Monitored by Single-Particle Tracking. Anal Chem. 2019, 91, 
13818-13823.

11 Rago, B.;Tumey, L. N.;Wei, C.;Barletta, F.;Clark, T.;Hansel, S.Han, X. Quantitative Conjugated Payload Measurement Using Enzymatic Release of Antibody-Drug Conjugate with Cleavable Linker. Bioconjug Chem.

2017, 28, 620-626.

12 Kern, J. C.;Cancilla, M.;Dooney, D.;Kwasnjuk, K.;Zhang, R.;Beaumont, M.;Figueroa, I.;Hsieh, S.;Liang, L.;Tomazela, D.;Zhang, J.;Brandish, P. E.;Palmieri, A.;Stivers, P.;Cheng, M.;Feng, G.;Geda, P.;Shah, S.;Beck, A.;Bresson, D.;Firdos, J.;Gately, D.;Knudsen, N.;Manibusan, A.;Schultz, P. G.;Sun, Y.Garbaccio, R. M. Discovery of Pyrophosphate Diesters as Tunable, Soluble, and Bioorthogonal Linkers for Site-Specific Antibody-Drug Conjugates. J Am Chem Soc. 2016, 138, 1430-1445.

13 Bessire, A. J.;Ballard, T. E.;Charati, M.;Cohen, J.;Green, M.;Lam, M. H.;Loganzo, F.;Nolting, B.;Pierce, B.;Puthenveetil, S.;Roberts, L.;Schildknegt, K.Subramanyam, C. Determination of Antibody-Drug Conjugate Released Payload Species Using Directed in Vitro Assays and Mass Spectrometric Interrogation. Bioconjug Chem. 2016, 27, 1645-1654.

14 Sang, H.;Lu, G.;Liu, Y.;Hu, Q.;Xing, W.;Cui, D.;Zhou, F.;Zhang, J.;Hao, H.;Wang, G.Ye, H. Conjugation site analysis of antibody-drug-conjugates (ADCs) by signature ion fingerprinting and normalized area quantitation approach using nano-liquid chromatography coupled to high resolution mass spectrometry. Anal Chim Acta.

2017, 955, 67-78.

15 Liu, Y.;Zhou, F.;Sang, H.;Ye, H.;Chen, Q.;Yao, L.;Ni, P.;Wang, G.Zhang, J. LC-MS/MS method for the simultaneous determination of Lys-MCC-DM1, MCC-DM1 and DM1 as potential intracellular catabolites of the antibody-drug conjugate trastuzumab emtansine (T-DM1). J Pharm Biomed Anal. 2017, 137, 170-177.

16 Doronina, S. O.;Mendelsohn, B. A.;Bovee, T. D.;Cerveny, C. G.;Alley, S. C.;Meyer, D. L.;Oflazoglu, E.;Toki, B. E.;Sanderson, R. J.;Zabinski, R. F.;Wahl, A. F.Senter, P. D. Enhanced activity of monomethylauristatin F through monoclonal antibody delivery: effects of linker technology on efficacy and toxicity. Bioconjug Chem. 2006, 17, 114-124.

17 Erickson, H. K.;Park, P. U.;Widdison, W. C.;Kovtun, Y. V.;Garrett, L. M.;Hoffman, K.;Lutz, R. J.;Goldmacher, V. S.Blattler, W. A. Antibody-maytansinoid conjugates are activated in targeted cancer cells by lysosomal degradation and linker-dependent intracellular processing. Cancer Res. 2006, 66, 4426-4433.

18 Dorywalska, M.;Strop, P.;Melton-Witt, J. A.;Hasa-Moreno, A.;Farias, S. E.;Galindo Casas, M.;Delaria, K.;Lui, V.;Poulsen, K.;Sutton, J.;Bolton, G.;Zhou, D.;Moine, L.;Dushin, R.;Tran, T. T.;Liu, S. H.;Rickert, M.;Foletti, D.;Shelton, D. L.;Pons, J.Rajpal, A. Site-Dependent Degradation of a Non-Cleavable Auristatin-Based Linker-Payload in Rodent Plasma and Its Effect on ADC Efficacy. PLoS One. 2015, 10, e0132282.

19 Cocchiaro, P.;De Pasquale, V.;Della Morte, R.;Tafuri, S.;Avallone, L.;Pizard, A.;Moles, A.Pavone, L. M. The Multifaceted Role of the Lysosomal Protease Cathepsins in Kidney Disease. Front Cell Dev Biol. 2017, 5, 114.

20 Schroder, B.Saftig, P. Intramembrane proteolysis within lysosomes. Ageing Res Rev. 2016, 32, 51-64. 
21 He, J.;Yu, S. F.;Yee, S.;Kaur, S.Xu, K. Characterization of in vivo biotransformations for trastuzumab emtansine by high-resolution accurate-mass mass spectrometry. MAbs. 2018, 10, 960-967.

22 Dong, L.;Li, C.;Locuson, C.;Chen, S.Qian, M. G. A Two-Step Immunocapture LC/MS/MS Assay for Plasma Stability and Payload Migration Assessment of Cysteine-Maleimide-Based Antibody Drug Conjugates. Anal Chem. 2018, 90, 5989-5994.

23 Niemikoski, H.;Koske, D.;Kammann, U.;Lang, T.Vanninen, P. Studying the metabolism of toxic chemical warfare agent-related phenylarsenic chemicals in vitro in cod liver. J Hazard Mater. 2020, 391, 122221.

24 Guo, J.;Wang, T.;Wu, T.;Zhang, K.;Yin, W.;Zhu, M.;Pang, Y.;Hao, C.;He, Z.;Cheng, M.;Liu, Y.;Zheng, J.;Gu, J.Zhao, D. Synthesis, bioconversion, pharmacokinetic and pharmacodynamic evaluation of $\mathrm{N}$-isopropyl-oxy-carbonyloxymethyl prodrugs of CZh-226, a potent and selective PAK4 inhibitor. Eur J Med Chem. 2020, 186, 111878.

25 Hyung, S. J.;Li, D.;Koppada, N.;Kaur, S.Saad, O. M. Method development of a novel PK assay for antibody-conjugated drug measurement of ADCs using peptide-linker drug analyte. Anal Bioanal Chem. 2019, 411, 2587-2596.

26 Huang, Y.;Del Nagro, C. J.;Balic, K.;Mylott, W. R., Jr.;Ismaiel, O. A.;Ma, E.;Faria, M.;Wheeler, A. M.;Yuan, M.;Waldron, M. P.;Peay, M. G.;Cortes, D. F.;Roskos, L.;Liang, M.Rosenbaum, A. I. Multifaceted Bioanalytical Methods for the Comprehensive Pharmacokinetic and Catabolic Assessment of MEDI3726, an Anti-Prostate-Specific Membrane Antigen Pyrrolobenzodiazepine Antibody-Drug Conjugate. Anal Chem. 2020.

27 Singh, A. P.;Guo, L.;Verma, A.;Wong, G. G.;Thurber, G. M.Shah, D. K. Antibody Coadministration as a Strategy to Overcome Binding-Site Barrier for ADCs: a Quantitative Investigation. AAPS J. 2020, 22, 28.

28 Wymant, J. M.;Sayers, E. J.;Muir, D.Jones, A. T. Strategic Trastuzumab Mediated Crosslinking Driving Concomitant HER2 and HER3 Endocytosis and Degradation in Breast Cancer. J Cancer. 2020, 11, 3288-3302.

29 Miao, R.;Li, M.;Zhang, Q.;Yang, C.Wang, X. An ECM-to-Nucleus Signaling Pathway Activates Lysosomes for C. elegans Larval Development. Dev Cell. 2020, 52, 21-37 e25.

30 Kissing, S.;Hermsen, C.;Repnik, U.;Nesset, C. K.;von Bargen, K.;Griffiths, G.;Ichihara, A.;Lee, B. S.;Schwake, M.;De Brabander, J.;Haas, A.Saftig, P. Vacuolar ATPase in phagosome-lysosome fusion. J Biol Chem. 2015, 290, 14166-14180.

31 Beck, A.;Goetsch, L.;Dumontet, C.Corvaia, N. Strategies and challenges for the next generation of antibody-drug conjugates. Nat Rev Drug Discov. 2017, 16, 315-337. 


\section{FIGURES}

Figure 1. An illustration of the in vitro TARSC approach that supports ADC quality assessment and mechanistic studies.
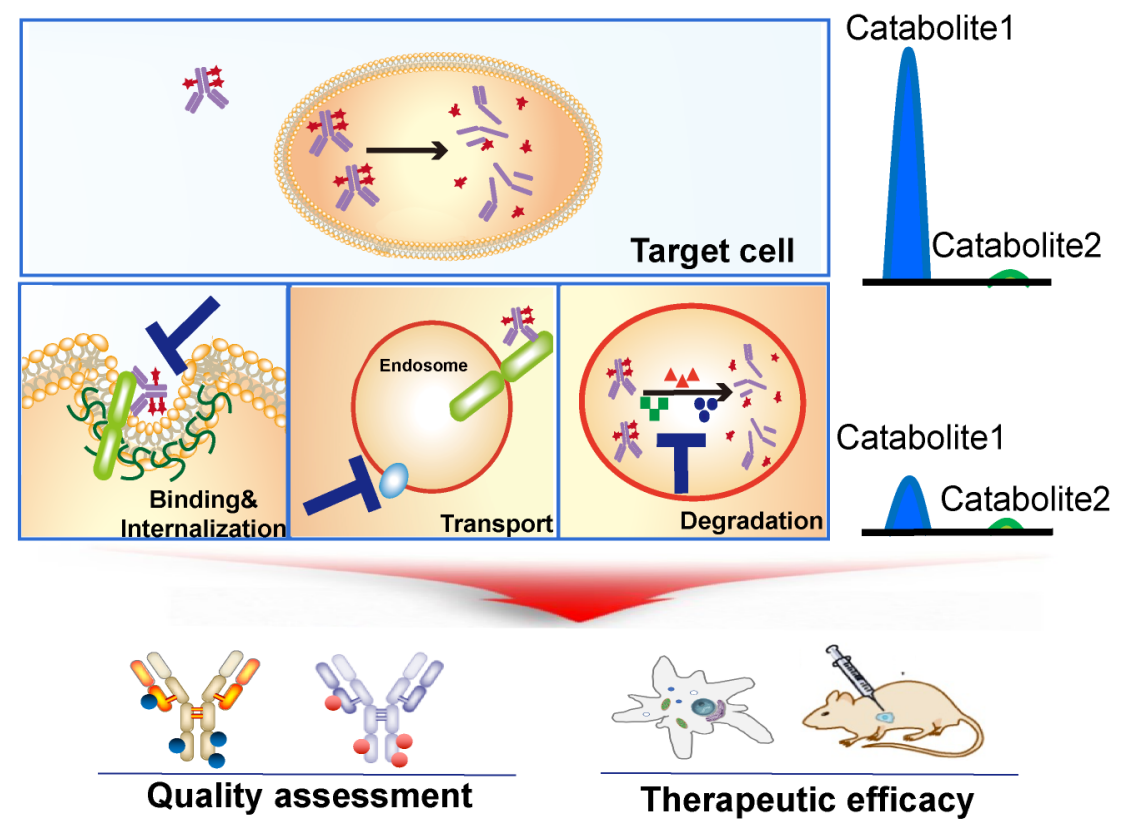
Figure 2. Lysosomal TARSC analysis of the commercial drug (CD) and biosimilar (BS) T-DM1s using rat liver crude lysosome fractions. (A) Catabolic kinetics of T-DM1 after incubation with the crude lysosome fractions isolated from rat liver ( $25 \mathrm{nM} \mathrm{CD}$ vs. BS T-DM1, $n=6$ ). (B) The areas under curve (AUCs) of the two major catabolites of the CD and BS T-DM1s were compared. (C) Scheme of lysosomal TARSC analysis conducted via the administration of specific inhibitors targeting different proteases. (D) Influence of given protease inhibitors $(10 \mu \mathrm{M}$ E64d, $10 \mu \mathrm{M}$ CA-074-ME, $20 \mu \mathrm{g} / \mathrm{mL}$ Aprotinin, $10 \mu \mathrm{g} / \mathrm{mL}$ pepstatin A) on the catabolic kinetics of the CD and BS T-DM1s $(n=6)$. All data are presented as mean \pm SEM. $* * \mathrm{P}<0.01$, One-way ANOVA.

A

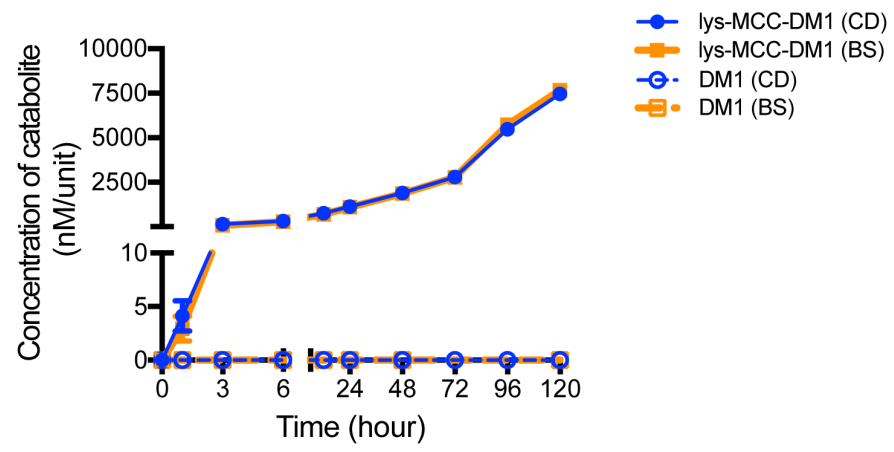

B

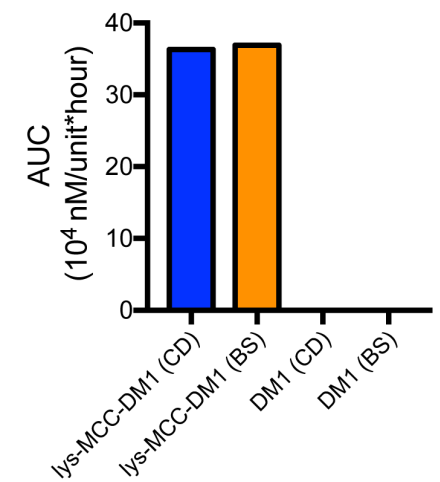

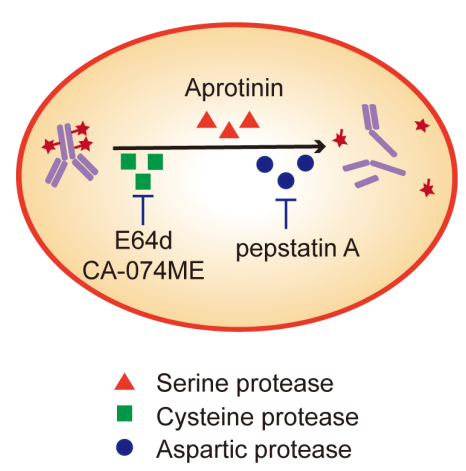

D

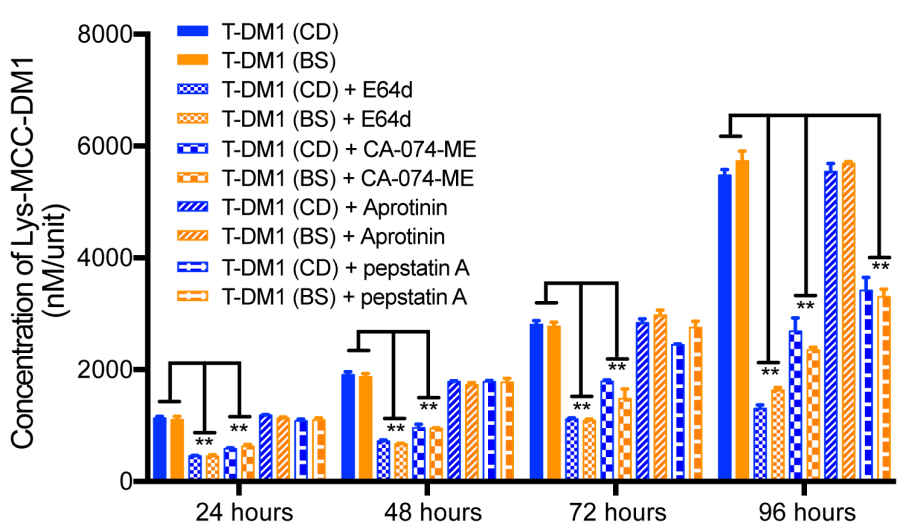


Figure 3. Catabolism profiles of the CD and BS T-DM1s in BT474 cells. (A) Time-resolved kinetic profiles of T-DM1 catabolites in BT474 cells administered with three different doses (12.5 nM, $25 \mathrm{nM}$ and $50 \mathrm{nM}$ ). (B) The areas under curve (AUCs) of the two major catabolites were compared. Data are expressed as mean $\pm \operatorname{SEM}(n=6)$.
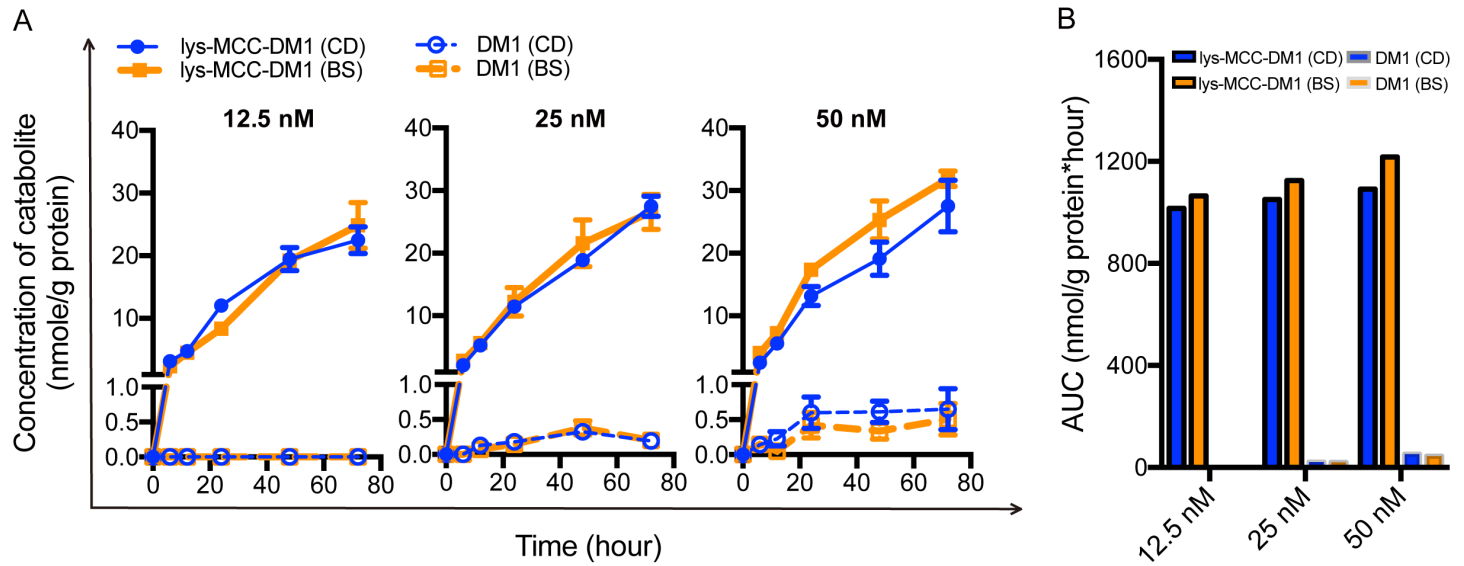
Figure 4. TARSC analysis determines the major catabolic proteins/pathway contributing to the payload release of the CD and BS T-DM1s in BT474 cells. (A)Transfection efficiency of HER2 siRNA in BT474 cells was visualized by immunofluorescence (Red, HER2; Blue, Hoechst) and measured by flow cytometry (n=6). (B) Impact of treatment with HER2 siRNA or negative control siRNA for 48 hours on production of the major T-DM1 catabolites for the CD and BS T-DM1s after incubation for another 72 hours. (C) Impact of inhibiting clathrin-dependent internalization with chlorpromazine (10 $\mu \mathrm{M})$ treatment for 2 hours on catabolites produced from the CD and BS T-DM1s, respectively. (D) Impact of inhibiting endosome-lysosome fusion with Bafilomycin A1 (1nM) treatment for 2 hours on catabolites produced from the CD and BS T-DM1s, respectively. (E) Impact of inhibiting cysteine, aspartic and serine protease-dependent proteolytic degradation by treatment with E64d $(20 \mu \mathrm{M})$, CA-074-ME $(3 \mu \mathrm{M})$, pepstatin A $(20 \mu \mathrm{g} / \mathrm{mL})$, aprotinin $(20$ $\mu \mathrm{g} / \mathrm{mL}$ ) for 72 hours on catabolites produced from the CD and BS T-DM1s in BT474 cells, respectively. The concentrations of the two catabolites, lys-MCC-DM1 and DM1, were quantified by LC-MS/MS ( $\mathrm{n}=6$ for each group). All data represent mean $\pm \mathrm{SEM}, * \mathrm{p}<0.05$, ** $\mathrm{p}<0.01, * * * \mathrm{p}<0.001$, Student's t-test.
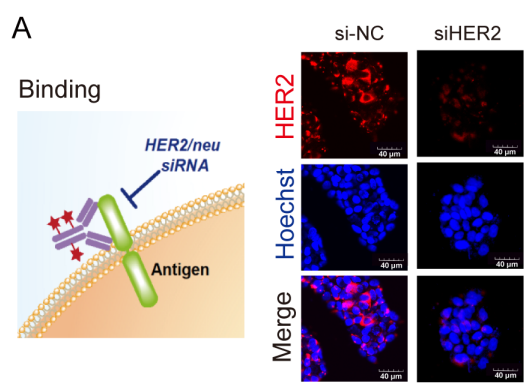

C

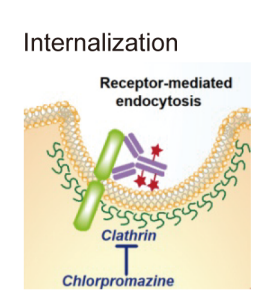

E

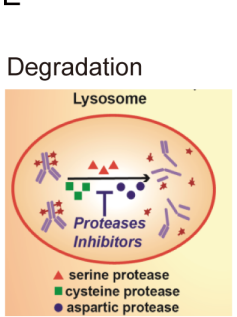

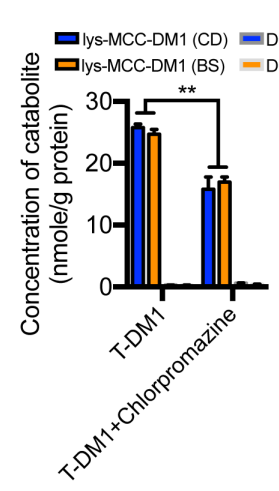
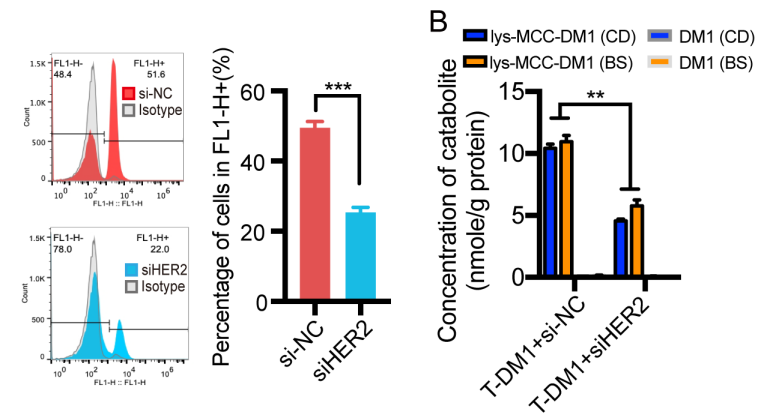

D
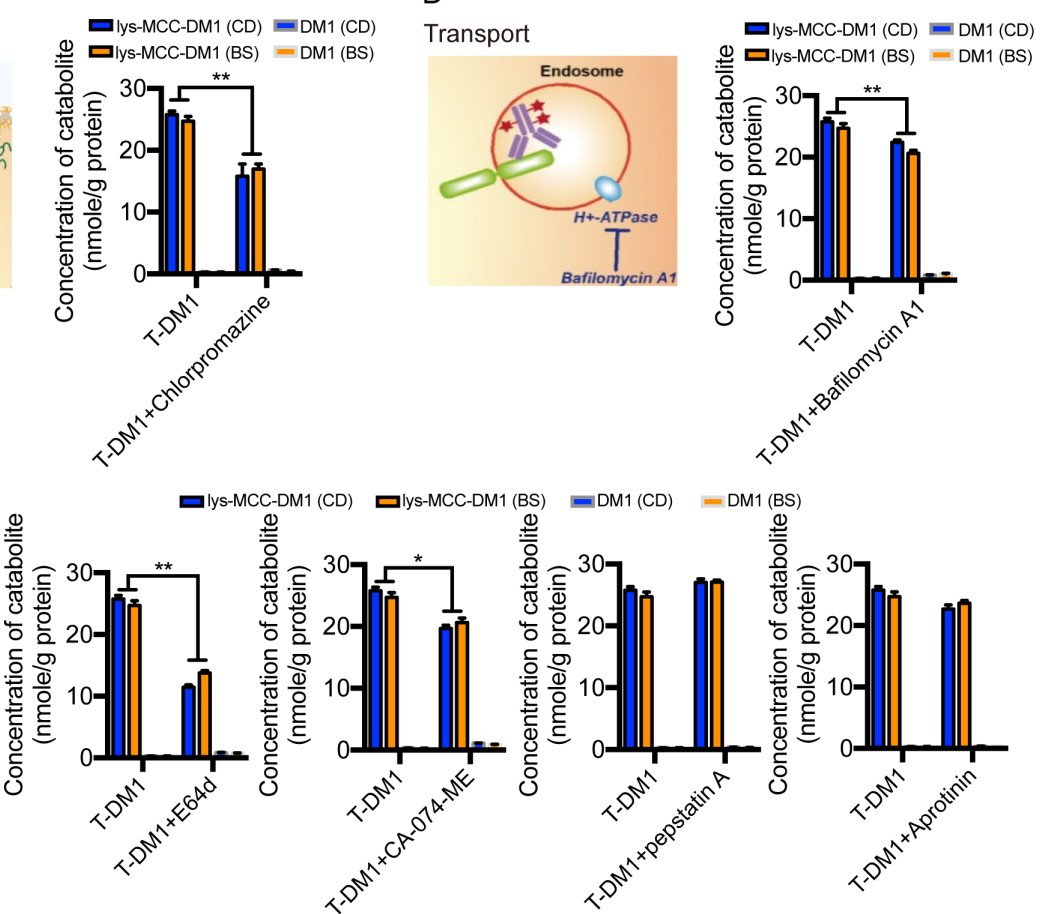
Figure 5. In vitro and in vivo therapeutic efficacy of the CD and BS T-DM1s. (A) Growth inhibition were measured at 120 hours post-administration $(n=6)$. (B) $\mathrm{IC}_{50}$ values were compared between the CD and BS T-DM1s. (C) Tumor volumes were measured every day and tumor growth curves were plotted for mice bearing BT474 cell-derived tumor xenografts. (D) AUCs of intra-tumoral lys-MCC-DM1 and DM1 measured from the CD and BS T-DM1-treated group, respectively. Specifically, 6 mice from each group were sacrificed at day 1 , day 4 , day 7 post-administration, and the tumors were collected to determine the catabolite concentration at each assayed time point for AUC calculation. All data represent mean $\pm \operatorname{SEM}(n=6)$. N.S., no significance, ${ }^{*} \mathrm{p}<0.05$, Student's t-test.

A

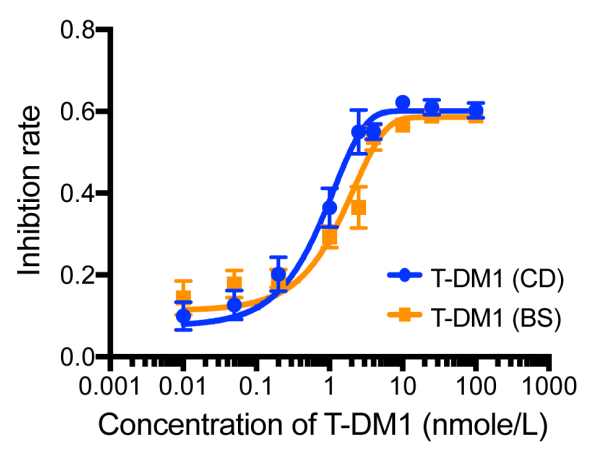

C

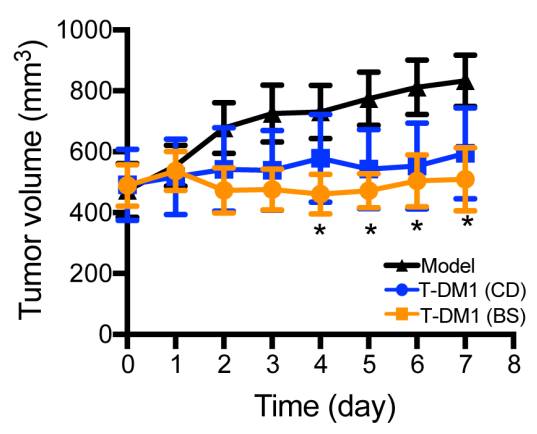

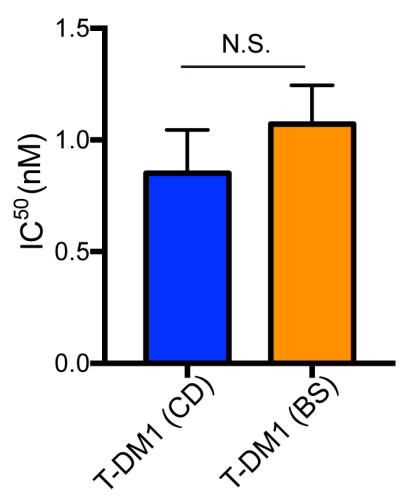

$\mathrm{D}$

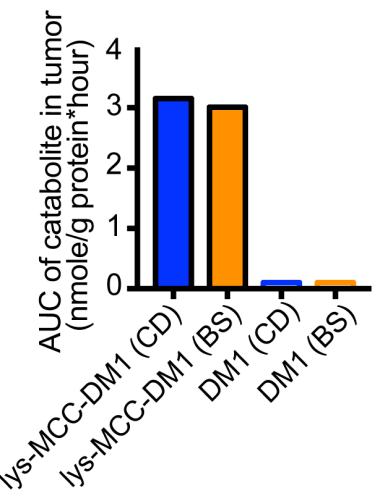


Figure 6. Catabolism profiles of cys-compound $T$ and compound $T$ produced from DX-006 after incubation with rat liver CLF. (A) Schematic illustration of the structure of DX-006. (B) Kinetic profiles of DX-006 catabolites after incubating DX-006 with the crude rat liver lysosomes for 1, 2, 4, 6, 12, 24, 48, 72, 96 and 120 hours. (C) AUCs of the detected DX-006 catabolites after incubation for 120 hours. (D) Lysosomal TARSC analysis of DX-006 achieved by co-administration DX-006 with specific cysteine proteases inhibitors (10 $\mu \mathrm{M} \mathrm{E64d,} 10 \mu \mathrm{M}$ CA-074-ME) for 48 hours, respectively. All data represent mean \pm SEM $(\mathrm{n}=3),{ }^{* *} \mathrm{p}<0.01, * * * \mathrm{p}<0.001$, One-way ANOVA.

A

compound T (Tub-006)

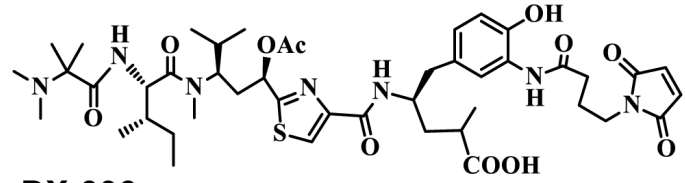

DX-006

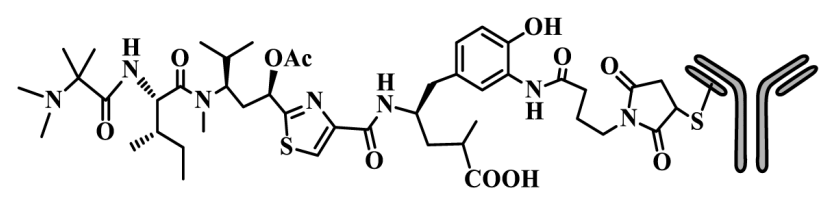

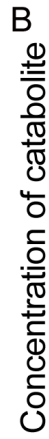

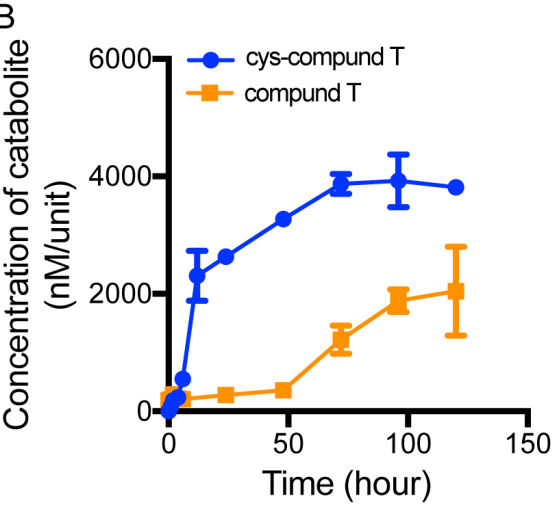

C

D

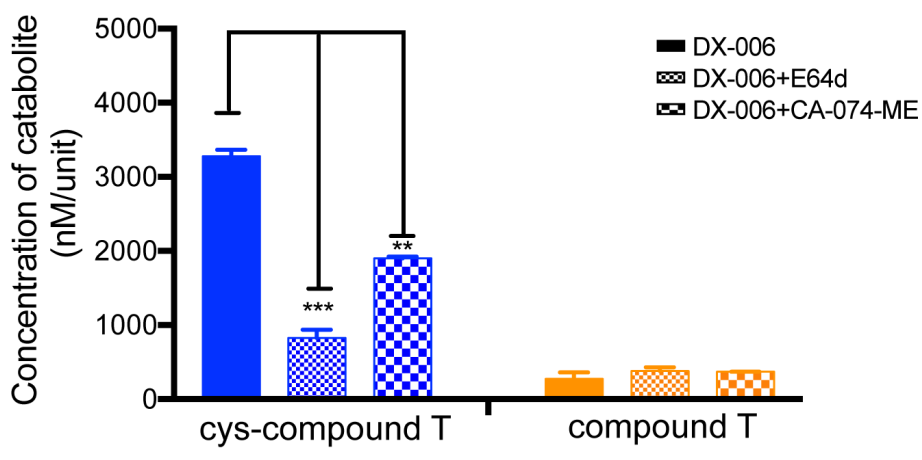

